\title{
Ivermectin as a novel complementary malaria control tool to reduce incidence and prevalence: a modelling study
}

Hannah C. Slater PhD (1)*, Prof Brian D. Foy PhD (2), Kevin Kobylinski PhD (3), Carlos Chaccour MD $(4,5,6)$, Oliver J. Watson MRes (1), Joel Hellewell PhD (1), Ghaith Aljayyoussi PhD (7), Teun Bousema $\operatorname{PhD}(8,9)$, Jeremy Burrows PhD (10), Prof Umberto D'Alessandro PhD (11), Haoues Alout PhD (12), Prof Feiko Ter Kuile PhD (7), Patrick G.T. Walker PhD (1), Prof Azra C. Ghani PhD (1), Menno R. Smit MD (7)

1) MRC Centre for Global Infectious Disease Analysis, Department of Infectious Disease Epidemiology, Imperial College London, London, UK

2) Arthropod-borne and Infectious Diseases Laboratory, Department of Microbiology, Immunology and Pathology, Colorado State University, Fort Collins, CO, USA

3) Department of Entomology, Armed Forces Research Institute of Medical Sciences, Bangkok, Thailand

4) ISGlobal, Hospital Clínic - Universitat de Barcelona, Barcelona, Spain

5) Instituto de Salud Tropical - Universidad de Navarra, Pamplona, Spain

6) Ifakara Health Institute, Ifakara, Tanzania

7) Liverpool School of Tropical Medicine (LSTM), Liverpool, UK

8) Department of Immunology and Infection, London School of Hygiene and Tropical Medicine, London, UK

9) Radboud Institute for Health Sciences, Radboud University Medical Centre, Nijmegen, The Netherlands

10) Medicines for Malaria Venture, Route de Pré Bois 20, 1215 Geneva 15, Switzerland

11) MRC Unit The Gambia at LSHTM, Fajara, Banjul, The Gambia

12) ASTRE unit (UMR117), INRA-CIRAD, Montpellier, France

*Corresponding author (hslater@path.org, PATH, 2201 Westlake Avenue, Seattle, WA, USA)

\section{Summary}

Background: Ivermectin has been identified as a potential new vector control tool to reduce malaria transmission. Mosquitoes feeding on a bloodmeal containing ivermectin have been shown to have a reduced lifespan, meaning they are less likely to live long enough to complete sporogony and become infectious.

Methods: In this study, we validate an existing population-level mathematical model of the impact of ivermectin on the mosquito population and malaria transmission to entomological and clinical data. The model is extended to include a range of complementary malaria interventions and to incorporate new data on higher doses with a longer mosquitocidal effect. We then simulate the impact of these doses in a range of usage scenarios in different transmission settings.

Findings: Mass drug administration (MDA) with ivermectin is predicted to reduce prevalence and incidence and is most effective in areas with a relatively short transmission season. In a highly seasonal moderate transmission setting, three rounds of ivermectin-only MDA spaced one month apart with a dose of $3 \times 300 \mu \mathrm{g} / \mathrm{kg}$ and $70 \%$ coverage is predicted to reduce clinical incidence by $71 \%$ and prevalence by $34 \%$ We predict that adding ivermectin MDA to seasonal malaria chemoprevention in this setting will reduce clinical incidence by an additional $77 \%$ in under 5 -year olds. Adding ivermectin MDA to MDA with antimalarials in this setting is predicted to reduce incidence by an additional $75 \%$. 
Interpretation: Ivermectin is a novel vector control tool that targets residual transmission, it has an excellent safety profile and has operationally synergistic distribution schedules with existing malaria interventions. Based on modelling predictions in this study, we propose that this drug could be a valuable addition to the malaria control toolbox, both in areas with persistently high transmission where existing vector control is insufficient and in areas approaching elimination to prevent resurgence.

Funding: Imperial College Junior Research Fellowship

\section{Research in context}

Evidence before this study

We searched PubMed and ScienceDirect on August 17th, 2019, for studies using mathematical models to assess the impact of ivermectin (to humans) on malaria prevalence and incidence, using the search terms "ivermectin" AND "malaria" AND ("modelling" OR "modeling"). The search was unrestricted by language or publication date. Using this search and by scanning reference lists of articles, we identified three publications in peer-reviewed journals. Slater et al. found that adding a single dose of ivermectin $200 \mathrm{\mu g} / \mathrm{kg}$ would only have a modest effect on reduction of malaria prevalence if distributed in mass drug administration (MDA) with dihydroartemisinin-piperaquine (DHA-P), although higher doses of ivermectin were predicted to have a greater and longer-lasting effect. However, a model developed by Stuckey and colleagues predicted that adding ivermectin to MDA with DHA-P in Zambia would have a negligible additional effect. Finally a theoretical mathematical model by Ngwa et al. predicts that treating symptomatic individuals with ivermectin would reduce the reproduction number of malaria.

Added value of this study

We present the first population-level mathematical model of the impact of ivermectin on the mosquito population and malaria transmission that has been validated to clinical and entomological field data. Furthermore, the model incorporates new empirical data on higher doses with a longer mosquitocidal effect and has been extended to assess ivermectin alone and in combination with a range of complementary malaria interventions, including mass drug administration and seasonal malaria chemoprevention. By simulating impact in a range of usage scenarios in different transmission settings, our study shows that mass drug administration with ivermectin is predicted to reduce prevalence and incidence and is most effective in areas with a relatively short transmission season. When used in combination with seasonal malaria chemoprevention or mass drug administration with antimalarials, we predict that ivermectin will increase and prolong the impact of these interventions.

Implications of all the available evidence

Our modelling results indicate that ivermectin alone, and to a greater extent when combined with antimalarial drugs, is predicted to have a major and prolonged effect on malaria prevalence and incidence in a range of transmission settings. We predict that adding ivermectin mass drug administration to current interventions can increase impact and help sustain reductions in transmission. Due to the operationally synergistic opportunities of co-administering ivermectin with other interventions that have the same distribution schedule (mass drug administration with antimalarials, and seasonal malaria chemoprevention), and the fact that ivermectin can directly target residual transmission that remains even with high coverage of long lasting insecticidal nets and indoor residual spraying with insecticides, we believe ivermectin is a powerful new tool which can complement existing malaria control efforts. 


\section{Introduction}

Despite increasing coverage of vector control (long lasting insecticidal nets (LLINS) and indoor residual spraying (IRS) with insecticides) and improved access to diagnosis and treatment, there were still an estimated 435,000 deaths from malaria in $2017^{1}$. Novel control methods targeting aspects of the transmission cycle currently missed by existing interventions may be needed to further reduce malaria burden. LLINs have contributed most to reductions in transmission ${ }^{2}$ but provide imperfect protection against human-vector contact, missing outdoor and early-biting mosquitoes. IRS targets only indoorfeeding and indoor-resting mosquitoes. Furthermore, there is evidence that mosquitoes are changing their behaviour to feed at times when people are not protected by these interventions ${ }^{3}$. Worryingly, insecticide resistance to the main chemicals has been reported worldwide ${ }^{4}$, resulting in reduced efficacy in killing mosquitoes.

IRS and LLINs will likely remain the cornerstones of malaria control but there is an urgent need for additional tools to supplement them. Several novel vector control approaches are being trialled ${ }^{5}$, including attractive targeted sugar baits ${ }^{6}$ and eave tubes ${ }^{7}$. Mosquitocidal drugs, such as the avermectin class of endectocides, are a potentially impactful novel approach to vector control. Endectocides work by killing mosquitoes that feed on humans or animals that have recently taken them. Ivermectin is the only drug in the class that is available for human use, and studies have shown that it is toxic to mosquitoes, delays refeeding ${ }^{8}$, reduces fecundity ${ }^{9}$ and locomotor activity ${ }^{10}$, and may inhibit sporozoite development ${ }^{11}$. Ivermectin has many attractive qualities as a novel malaria control tool. Unlike IRS and LLINs, it targets mosquitoes regardless of feeding location or time. It can be given to cattle, so could be dual-administered to both humans and cattle in areas with zoophilic malariatransmitting mosquitoes ${ }^{12}$. Furthermore, it has a novel model of action, reducing the likelihood of cross-resistance with existing insecticides ${ }^{9}$.

Mass ivermectin administration could be combined in an operationally opportunistic manner with current interventions already being carried out on a large scale across malaria endemic regions. Single dose mass drug administration (MDA) with ivermectin (and other anti-helminthic drugs) is carried out to control neglected tropical diseases (NTDs) across Africa ${ }^{13}$ - extending the dosing schedule and frequency of administration in line with the malaria transmission season could have an impact on malaria transmission. Seasonal malaria chemoprevention (SMC), the monthly distribution of antimalarial drugs to children 3-59 months old during the peak months of transmission is being implemented in 12 countries in the Sahel region of Africa ${ }^{1}$. Combining SMC with population-wide ivermectin distribution could further protect children from being re-infected and reduce malaria transmission. Finally, MDA with antimalarials has been trialled in several malaria endemic countries to either accelerate toward elimination ${ }^{14}$, reduce malaria burden ${ }^{15}$ or contain the spread of artemisinin resistant parasites through local elimination ${ }^{16}$; ivermectin could be combined with this intervention to increase and prolong impact.

The doses of ivermectin typically used for onchocerciasis and lymphatic filariasis control (singe doses of $150-200 \mu \mathrm{g} / \mathrm{kg}$ ) have a short mosquitocidal effect of around 5-6 days ${ }^{17}$ and limited impact on mosquito populations ${ }^{18}$ and transmission unless distributed frequently ${ }^{19}$. Recent clinical trials investigating the impact of higher doses have produced promising results. Three doses of $300 \mu \mathrm{g} / \mathrm{kg}$ given over three days has a mosquitocidal effect in humans for 28 days against Anopheles gambiae s.s. ${ }^{20}$ and a single dose of $400 \mu \mathrm{g} / \mathrm{kg}$ was effective for at least ten days against Anopheles minimus and 6-10 days against Anopheles dirus, two of the most important malaria vectors in Southeast Asia ${ }^{21,22}$. A slow-release ivermectin implant has achieved mosquitocidal concentrations for 40 weeks in cattle ${ }^{12}$, and a novel slow-release, gastric-resident, drug delivery technology in development has achieved 
mosquitocidal concentrations of ivermectin for around 14 days in pigs ${ }^{23}$. Fluralaner and afoxolaner, two drugs from the isoxazolines class of endectocides used in veterinary medicine, have also been shown to be toxic to mosquitoes. Preliminary estimates indicate that they could remain at effective mosquitocidal concentrations for 50-90 days, but have not yet been tested for safety in humans ${ }^{24}$ and regulatory approval for human use may take up to a decade ${ }^{25}$. The combination of non-ivermectin mosquitocidal drugs administered to cattle alongside ivermectin administered to humans has been suggested as an approach to simultaneously target anthropophilic and zoophilic mosquitoes whilst preventing the development of resistance to ivermectin via a dual-chemistry approach which is recommended in other forms of vector control ${ }^{26}$.

The growing body of evidence that higher doses of ivermectin have a prolonged efficacious duration, as well as the development of other slow release or long lasting endectocides has led to calls to better understand the potential impact of these drugs on malaria transmission ${ }^{17}$. In this study, we use a mathematical model to estimate the impact of ivermectin MDA and to provide guidance on the potential scenarios in which they could complement existing malaria interventions to further reduce malaria transmission and burden.

\section{Methods}

We previously developed a malaria transmission model ${ }^{27}$ to capture the impact of ivermectin ${ }^{28}$ on vector survival. Here we extend the model to: i) incorporate a range of complementary malaria interventions, ii) allow a wider range of mosquitocidal drug profiles, iii) track the parity rate of vector populations, and iv) allow for correlation between who receives drugs each round in mass administration interventions.

\section{Malaria transmission model}

The deterministic compartmental model incorporates transmission between mosquito and human hosts $^{27,29}$. Individuals begin life susceptible with a level of maternally-acquired immunity which quickly wanes. Upon inoculation with an infectious bite they either become infected (with probability determined by their level of pre-erythrocytic immunity), whereupon they either develop clinical disease or asymptomatic infection (determined by their levels of blood-stage immunity). Individuals with clinical disease have a probability of being successfully diagnosed and treated. Treated individuals are prophylactically protected for a duration based on the properties of the antimalarial taken. Untreated individuals with clinical disease are assumed to have symptomatic infection for an average 5 days before transitioning to becoming asymptomatically infected. Asymptomatically infected individuals remain infected for an average 310 days $^{27}$, but their probability of being detectable by microscopy decreases over the course of the infection to capture the effect of decreasing parasite densities. Individuals that are susceptible or have asymptomatic infection can be superinfected which follows the same infection process. The acquisition and loss of immunity is dynamically modelled and determines the probability of infection, the probability of developing symptoms and the detectability and transmissibility of infection. Transmission from mosquitoes to humans is determined by the entomological inoculation rate, which is a product of the mosquito biting rate, sporozoite rate, functions determining the relative biting rate on different subgroups (capturing heterogeneity in exposure) and age and the probability of successful inoculation. Similarly, transmission from humans to mosquitoes is determined by the infectivity of the human, which is based on their infection state, the mosquito biting rate, the age- and heterogeneity-biting rates and the probability of successful infection. We assume a constant and isolated population, with no movement of infected humans or mosquitoes in or out of the intervention area. Details of the model are provided in the Appendix, page 1-11. 


\section{Intervention models}

We assess the impact of ivermectin MDA by assuming that a proportion of bloodmeals taken by mosquitoes contain ivermectin (determined by the coverage of ivermectin in the human population). Mosquitoes ingesting ivermectin transition to a new compartment where they experience an elevated mortality rate for the rest of their life. The 'ivermectin-fed' compartments are tracked for each day post ivermectin-administration, each with a unique mortality rate to capture the elevated but waning mosquitocidal effect of ivermectin over time as the concentration in human blood decreases. The elevated mortality rates are estimated using a pharmacokinetic (PK)-pharmacodynamic (PD) model ${ }^{30}$ which has been fitted to human ivermectin plasma concentrations and corresponding mosquito mortality data from feeding experiments conducted using Anopheles gambiae sensu stricto ${ }^{20}$. Delayed refeeding, reduced egg laying and reduced sporogonic development are not explicitly modelled as these effects are minimal compared to the mosquitocidal effects ${ }^{28}$. We also incorporate the impact of existing malaria interventions - including LLINs, SMC and MDA - using existing intervention model ${ }^{29}$.

\section{Model validation}

The model is validated against data from two ivermectin trials: a study across three countries consisting of a single round of ivermectin MDA and focusing on entomological data ${ }^{18}$, and a cluster randomised trial (CRT) conducted in Burkina Faso consisting of a single round of ivermectin MDA in the control arm and six rounds of ivermectin MDA in the intervention arm and focusing on clinical incidence in a cohort of children $\leq 5$ years old ${ }^{19}$. This model validation is presented in the Appendix, pages 12-17.

\section{Intervention Scenarios}

We explore the potential impact of ivermectin on malaria prevalence and clinical incidence for the scenarios shown in Box 1 . The scenarios are simulated in three seasonality 'archetypes' that encapsulate the range of transmission in sub-Saharan Africa ${ }^{31}$ : i) highly seasonal, based on Fatick in Senegal, with a transmission season of approximately 4 months, ii) seasonal, based on Bougouriba in Burkina Faso, with a season of 7-8 months, and iii) perennial, based on Equateur in Democratic Republic of Congo (DRC), with year-round transmission. Unless stated otherwise, all simulations have a mean annual all-age slide prevalence of $30 \%$.

Ivermectin is recommended for all individuals $>15 \mathrm{~kg} / \geq 90 \mathrm{~cm}$, however, for simplicity we assume all children $<59$ months are below this threshold, and all children $\geq 59$ months are above this threshold. Coverage of ivermectin is defined using the number of all individuals $\geq 5$ years old as the denominator. Ivermectin is also not recommended for pregnant women, which is why we only consider modest coverage estimates (maximum of $70 \%$ ).

We consider two ivermectin regimens: a single dose of $400 \mu \mathrm{g} / \mathrm{kg}(1 \times 400)$ and three consecutive daily doses of $300 \mu \mathrm{g} / \mathrm{kg}$ per day $(3 \times 300)$. The former is the highest dose currently recommended for lymphatic filariasis MDAs, and the latter is the dose that was viewed as most promising in a recent clinical trial ${ }^{20}$.

For all scenarios the intervention is introduced optimally in relation to the location-specific seasonality profile of each simulation, obtained by simulating the model at different start times and selecting the time that results in the greatest reduction in cumulative incidence. 


\section{Role of Funding Source}

The sponsor of the study had no role in study design, data collection, data analysis, data interpretation, or writing of the report. The corresponding author had full access to all the data in the study and had final responsibility for the decision to submit for publication.

\section{Results}

The estimated pharmacokinetic profiles of the two dosing regimens are shown in Figure 1a. These were combined with the mosquito hazard ratios estimated in Smit et al. ${ }^{20}$ for each time after ivermectin administration that mosquitoes were fed on the treated individuals' blood (day $2+4$ hours, $\mathrm{d} 7, \mathrm{~d} 10, \mathrm{~d} 14, \mathrm{~d} 21, \mathrm{~d} 28$ ) to estimate the relationship between drug concentration and the mosquito hazard ratio (Figure 1b). Figures $1 \mathrm{a}-\mathrm{b}$ were then combined to estimate the daily hazard of mortality of mosquitoes biting each day (Figure 1c).

\section{Ivermectin only MDA}

We first simulated the impact of ivermectin only, assuming all other interventions continued at their current coverage levels (Figure 2, Table 1). The impact of ivermectin is predicted to be greatest in the setting with the shortest transmission season, with a predicted reduction in clinical incidence of $62 \%$ and $71 \%$ for the $1 \times 400 \mu \mathrm{g} / \mathrm{kg}$ and $3 \times 300 \mu \mathrm{g} / \mathrm{kg}$ doses respectively. The intervention is predicted to be less effective in areas with perennial transmission, where the equivalent reductions are $28 \%$ and $31 \%$ respectively. We predict that ivermectin has a greater impact on incidence than on prevalence. This is because the intervention prevents new infections by killing infected and infectious mosquitoes rather than clearing older asymptomatic infections. The sensitivity of these results to the impact of repeat ivermectin distribution assuming different durations and magnitudes of mosquitocidal effect (including a hypothetical mosquitocidal drug with a 90-day efficacious period), population coverage levels, number of rounds and timing between rounds and transmission intensities, and exploring the impact of importation of infected individuals into the intervention area, as well as synergies between interventions is shown in the Appendix, pages 18-28.

\section{Ivermectin MDA with SMC}

Figure 3 and Table 1 show the estimated impact of population-wide ivermectin MDA in combination with SMC Adding ivermectin MDA is predicted to increase the reduction in clinical cases in children $<5$ years old compared to SMC alongside existing core interventions. In a highly seasonal setting, we predict that SMC alone reduces clinical cases by $58 \%$ but adding population-wide ivermectin MDA increases this figure to $87 \%(1 \times 400 \mu \mathrm{g} / \mathrm{kg})$ or $90 \%(3 \times 300 \mu \mathrm{g} / \mathrm{kg})$ in the year after the start of the intervention. This corresponds to an incremental impact on top of SMC alone of $69 \%$ and $77 \%$, respectively. The reduction in clinical incidence is predicted to be lower in a setting with a longer transmission season. Here, the incremental impact of ivermectin is $51 \%(1 \times 400 \mu \mathrm{g} / \mathrm{kg})$ and $58 \%$ $(3 \times 300 \mu \mathrm{g} / \mathrm{kg})$. The impact of expanding SMC distribution to all individuals under the age of 10 (alongside ivermectin to individuals over the age of 10) is shown in the Appendix, pages 25-26.

Delivering SMC to children $<5$ years old and ivermectin MDA population-wide ( $\geq 5$ years old) is also predicted to have a dramatic impact on population level prevalence - whereas SMC alone is predicted 
to reduce all-age prevalence by only $19-21 \%$, adding ivermectin ( $1 \times 400 \mu \mathrm{g} / \mathrm{kg}$ dose) is predicted to reduce all-age prevalence by $52 \%$ (highly seasonal setting) or $45 \%$ (seasonal setting).

\section{Ivermectin and DHA-P MDA for burden reduction}

The impact of MDA with DHA-P and ivermectin for burden reduction in a highly seasonal moderate transmission setting with three rounds spaced one month apart is shown in Fig. 4a,d, and in a perennial moderate transmission setting with three rounds spaced one month apart in Fig. 4b,e or three rounds spaced four months apart in Fig. 4 c,f. MDA with DHA-P and ivermectin is predicted to be most effective in a seasonal transmission setting; predicted reduction in clinical incidence is $91 \%$

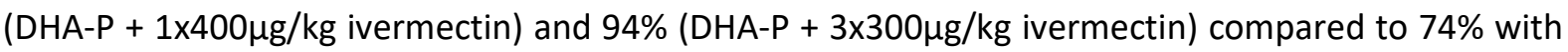
DHA-P alone (Table 1). In a perennial setting, a greater reduction in burden in achieved by spacing the rounds evenly throughout the year - in this scenario, the incremental impact of ivermectin in addition to DHA-P is also greater (Table 1).

\section{Ivermectin and DHA-P MDA for elimination}

Figure 5 shows the impact of MDA with DHA-P and ivermectin in a seasonal low transmission setting. Here adding ivermectin to DHA-P prevents the rebounds in transmission between rounds and is predicted to prolong the overall impact of the MDA intervention.

\section{Discussion}

Our modelling results predict that ivermectin alone, and to greater extent when combined with antimalarial drugs, could have a major and prolonged effect on malaria prevalence and incidence across a range of transmission settings. We predict that adding ivermectin MDA to current interventions can increase impact and sustain reductions in transmission. Due to the operationally synergistic opportunities of co-administering ivermectin with other interventions that have the same distribution schedule (MDA with antimalarials, SMC), and the fact that ivermectin can directly target residual transmission that remains even with high coverage of vector control, ivermectin may be a promising new complementary malaria tool.

In a seasonal setting, adding ivermectin MDA to SMC has a greater incremental impact on reducing prevalence and incidence compared to adding ivermectin MDA to DHA-P MDA, however the total impact of the latter intervention is greater. During SMC, a large proportion of the population remain untreated and unprotected, therefore adding an intervention that reduces the infectious vector population means that these individuals will also receive a benefit. However, MDA with DHA-P provides prophylaxis to a larger proportion of the population, so reducing the infectious vector population with ivermectin has a lower additional impact as a large proportion cannot be re-infected anyway.

SMC is widely conducted, extremely effective, and a key intervention in in the Sahel region which experiences some of the highest rates of malaria worldwide. We predict that administering ivermectin to the population $\geq 5$ years old could not only increase the impact of SMC in children under 5 , but could also increase the population-level benefit, reducing clinical incidence across the whole population.

Our results suggest that the $3 \times 300 \mu \mathrm{g} / \mathrm{kg}$ dose is only marginally more impactful than the $1 \times 400 \mu \mathrm{g} / \mathrm{kg}$ dose. Although the hazard-ratio area under the curve (and above 1) is $78 \%$ greater for $3 \times 300 \mu \mathrm{g} / \mathrm{kg}$ compared to $1 \times 400 \mu \mathrm{g} / \mathrm{kg}$ (Figure 1c), the highly non-linear effect of increased mortality on the proportion of mosquitoes completing sporogony and becoming infectious means that the duration the hazard ratio is above some threshold is more important that the magnitude of the hazard ratio. Even for a hazard ratio of 2 , the proportion of mosquitoes surviving long enough to complete 
sporogony is $63 \%$ lower than in the absence of ivermectin. The hazard ratio is $>2$ for 14 days with $3 \times 300 \mu \mathrm{g} / \mathrm{kg}$ and for 10 days with $1 \times 400 \mu \mathrm{g} / \mathrm{kg}$. The difference between the two regimens is greater in a highly-seasonal compared to a perennial setting (Figure 2) because, with the former, ivermectin's effective window covering a greater proportion of annual transmission.

A key assumption in the ivermectin component of the model is that the hazard ratios observed in a clinical laboratory setting can be applied to the known mortality rates of wild mosquitoes. For example, we assume that a mosquito dying twice as quickly in the laboratory after a certain dose of ivermectin would also die twice as quickly in the wild, albeit with a considerably higher baseline mortality rate. In the model, we assume that the mean baseline lifespan of an Anopheles gambiae mosquito is 10 days in the wild whereas the lifespan of mosquitoes in laboratory experiments is around $14-30$ days $^{20,32}$.

The model accurately captures changes in entomological outcomes observed in the field; however, these field data are limited (Appendix, page 14). Future entomology data collected in CRTs is therefore needed to validate or refine this assumption. The results presented here assume all mosquitoes are Anopheles gambiae s.s., however, there is no evidence that other African vectors would be less sensitive ${ }^{12,32,33}$.

Further limitations include that the $3 \times 300 \mu \mathrm{g} / \mathrm{kg}$ hazard estimates were derived directly from data ${ }^{13}$ whereas the $1 \times 400 \mu \mathrm{g} / \mathrm{kg}$ hazard ratios were estimated using a PK-PD model. The data used to derive both sets of hazard ratios were from a trial where ivermectin was co-administered with DHA-P. Preliminary data suggest an interaction between these drugs that increases ivermectin bioavailability, peak concentration, and mosquito killing effect compared to that of ivermectin alone ${ }^{21}$. Additionally, it remains to be determined whether the observed effect of ivermectin solely reflects that of the parent compound, or whether there is also an active ivermectin metabolite with mosquitocidal properties $^{21}$.

The results presented here assume a constant and isolated population, with no movement of infected humans or vectors into or out of the intervention area. Although in a sensitivity analysis (see Appendix, pages 24-25) we did not find a major impact of this assumption, further exploration of the effect of this intervention in models that capture spatial linkage between populations is warranted.

To provide empirical evidence for the utility of ivermectin across different settings CRTs are needed. An earlier small CRT of repeated ivermectin MDA in Burkina Faso ${ }^{19}$ found a $19.6 \%$ reduction in episodes of clinical incidence in a cohort of children $\leq 5$ years old (Appendix, pages 15-17). Whilst the $1 \times 400 \mu \mathrm{g} / \mathrm{kg}$ dose has been used for lymphatic filariasis control, the $3 \times 300 \mu \mathrm{g} / \mathrm{kg}$ dose has never been delivered at scale to whole populations. An ongoing CRT in The Gambia (NCT03576313) will provide evidence on the safety and acceptability of this higher dose $(3 \times 300 \mu \mathrm{g} / \mathrm{kg})$ when given in combination with DHA-P. Questions remain surrounding the feasibility of delivering this intervention at scale and the implications and potential adherence issues of treating populations with a drug that may provide them no direct benefits (if they do not have any other infections that ivermectin treats).

The appetite from national malaria control programs and funders to implement ivermectin MDA still needs to be ascertained. These decisions will depend in part on estimates of cost-effectiveness in comparison to other malaria interventions, particularly other novel vector control tools that might be targeted in areas with high transmission and high coverage of existing vector control tools. Mass ivermectin distribution in Loaisis-endemic regions may require a test-and-not-treat strategy, as it can cause adverse events in Loa loa infected individuals ${ }^{34}$. 
New longer lasting ivermectin formulations ${ }^{12,23}$ or other mosquitocidal drugs ${ }^{24}$ offer a promising new opportunity for malaria control, however, the benefit of current formulations of ivermectin should not be underplayed. Ivermectin is known to be safe and accepted by communities who have received MDAs for decades as part of the control of lymphatic filariasis and onchocerciasis. Ongoing CRTs using ivermectin will provide an opportunity to evaluate the impact of mosquitocidal drugs and provide evidence to guide decision making for both current and new longer lasting versions of these drugs.

As of September 2019, in addition to the ivermectin CRT underway in The Gambia, there are four more trials (that we are aware of) planned to start in 2019 or 2020. These trials are being conducted in different transmission settings with different doses and distribution schedules, different malaria vectors, and different coverages of other interventions. Models that have been validated against clinical and entomological data, such as the one presented here, will offer a useful way to compare results from these diverse trials, to synthesise evidence, and provide a robust framework to extrapolate from these trials to wider-scale predictions. 


\section{Disclaimers}

Material has been reviewed by the Walter Reed Army Institute of Research. There is no objection to its presentation and/or publication. The opinions or assertions contained herein are the private views of the author, and are not to be construed as official, or as reflecting true views of the Department of the Army or the Department of Defense.

ISGlobal is a member of the CERCA Programme, Generalitat de Catalunya

\section{Funding Sources}

This work was funded by an Imperial College Junior Research Fellowship to HCS with additional financial support from the Bill and Melinda Gates Foundation.

HCS and CC received additional financial support from Bill and Melinda Gates Foundation under the Ivermectin development roadmap (OPPP1177135) and from Unitaid under the Project Preparation Facility of the BOHEMIA grant (2018-30-ISG)

\section{Contributors}

HCS performed the transmission modelling analysis and processed all model outputs. HCS, BDF, KK, CC, TB, PGTW, MRS designed the analysis. GA produced the PK/PD modelling outputs. BDF, KK, HA, FTK, MRS collected data. HCS, OJW, JH developed the model code. HCS wrote the first draft of the manuscript. All authors contributed to writing and editing the manuscript. All authors approved the final manuscript.

\section{References}

1 Organization, W. H. World malaria report 2018. (2018).

2 Bhatt, S. et al. The effect of malaria control on Plasmodium falciparum in Africa between 2000 and 2015. Nature 526, 207, doi:10.1038/nature15535 https://www.nature.com/articles/nature15535\#supplementary-information (2015).

3 Moiroux, N. et al. Changes in Anopheles funestus Biting Behavior Following Universal Coverage of Long-Lasting Insecticidal Nets in Benin. The Journal of Infectious Diseases 206, 1622-1629, doi:10.1093/infdis/jis565 (2012).

4 Knox, T. B. et al. An online tool for mapping insecticide resistance in major Anopheles vectors of human malaria parasites and review of resistance status for the Afrotropical region. Parasites \& Vectors 7, 76, doi:10.1186/1756-3305-7-76 (2014).

5 Barreaux, P. et al. Priorities for Broadening the Malaria Vector Control Tool Kit. Trends in Parasitology 33, 763-774, doi:https://doi.org/10.1016/j.pt.2017.06.003 (2017). 
Beier, J. C., Müller, G. C., Gu, W., Arheart, K. L. \& Schlein, Y. Attractive toxic sugar bait (ATSB) methods decimate populations of Anopheles malaria vectors in arid environments regardless of the local availability of favoured sugar-source blossoms. Malaria Journal 11, 31, doi:10.1186/1475-2875-11-31 (2012).

Sternberg, E. D. et al. Eave tubes for malaria control in Africa: initial development and semifield evaluations in Tanzania. Malaria Journal 15, 447, doi:10.1186/s12936-016-1499-8 (2016).

8 Kobylinski, K. C. et al. The effect of oral anthelmintics on the survivorship and re-feeding frequency of anthropophilic mosquito disease vectors. Acta Tropica 116, 119-126, doi:https://doi.org/10.1016/j.actatropica.2010.06.001 (2010).

9 Foy, B. D., Kobylinski, K. C., Silva, I. M. d., Rasgon, J. L. \& Sylla, M. Endectocides for malaria control. Trends in Parasitology 27, 423-428, doi:https://doi.org/10.1016/i.pt.2011.05.007 (2011).

10 Sampaio, V. d. S. et al. What does not kill it makes it weaker: effects of sub-lethal concentrations of ivermectin on the locomotor activity of Anopheles aquasalis. Parasites \& Vectors 10, 623, doi:10.1186/s13071-017-2563-0 (2017).

11 Kobylinski, K. C., Foy, B. D. \& Richardson, J. H. Ivermectin inhibits the sporogony of Plasmodium falciparum in Anopheles gambiae. Malaria Journal 11, 381, doi:10.1186/14752875-11-381 (2012).

12 Chaccour, C. J. et al. Targeting cattle for malaria elimination: marked reduction of Anopheles arabiensis survival for over six months using a slow-release ivermectin implant formulation. Parasites \& Vectors 11, 287, doi:10.1186/s13071-018-2872-y (2018).

13 Hotez, P. J. Mass Drug Administration and Integrated Control for the World's High-Prevalence Neglected Tropical Diseases. Clinical Pharmacology \& Therapeutics 85, 659-664, doi:10.1038/clpt.2009.16 (2009).

14 Eisele, T. P. et al. Short-term Impact of Mass Drug Administration With Dihydroartemisinin Plus Piperaquine on Malaria in Southern Province Zambia: A Cluster-Randomized Controlled Trial. The Journal of Infectious Diseases 214, 1831-1839, doi:10.1093/infdis/jiw416 (2016).

15 Tiono, A. B. et al. A controlled, parallel, cluster-randomized trial of community-wide screening and treatment of asymptomatic carriers of Plasmodium falciparum in Burkina Faso. Malaria Journal 12, 79, doi:10.1186/1475-2875-12-79 (2013).

16 Tripura, R. et al. A Controlled Trial of Mass Drug Administration to Interrupt Transmission of Multidrug-Resistant Falciparum Malaria in Cambodian Villages. Clinical Infectious Diseases 67, 817-826, doi:10.1093/cid/ciy196 (2018).

17 Chaccour, C. J. et al. Establishment of the Ivermectin Research for Malaria Elimination Network: updating the research agenda. Malaria Journal 14, 243, doi:10.1186/s12936-0150691-6 (2015).

18 Alout, H. et al. Evaluation of ivermectin mass drug administration for malaria transmission control across different West African environments. Malaria Journal 13, 417, doi:10.1186/1475-2875-13-417 (2014).

19 Foy, B. et al. Repeat ivermectin mass drug administrations for control of malaria (RIMDAMAL): a cluster-randomized trial to evaluate efficacy and risk of harms. Lancet ((in press)).

20 Smit, M. R. et al. Safety and mosquitocidal efficacy of high-dose ivermectin when coadministered with dihydroartemisinin-piperaquine in Kenyan adults with uncomplicated malaria (IVERMAL): a randomised, double-blind, placebo-controlled trial. The Lancet Infectious Diseases 18, 615-626, doi:https://doi.org/10.1016/S1473-3099(18)30163-4 (2018).

21 Kobylinski, K. et al. Pharmacokinetic and pharmacodynamic properties of ivermectin: Ivermectin for Malaria in Southeast Asia (IMSEA), Thailand. ASTMH (2017).

22 Kobylinski, K. C. et al. Ivermectin susceptibility and sporontocidal effect in Greater Mekong Subregion Anopheles. Malaria Journal 16, 280, doi:10.1186/s12936-017-1923-8 (2017). 
23 Bellinger, A. M. et al. Oral, ultra-long-lasting drug delivery: Application toward malaria elimination goals. Science Translational Medicine 8, 365ra157 (2016).

24 Miglianico, M. et al. Repurposing isoxazoline veterinary drugs for control of vector-borne human diseases. Proceedings of the National Academy of Sciences (2018).

Burrows, J. et al. A discovery and development roadmap for new endectocidal transmissionblocking agents in malaria. Malaria Journal 17, 462, doi:10.1186/s12936-018-2598-5 (2018). Ranson, H. \& Lissenden, N. Insecticide Resistance in African Anopheles Mosquitoes: A Worsening Situation that Needs Urgent Action to Maintain Malaria Control. Trends in Parasitology 32, 187-196, doi:https://doi.org/10.1016/i.pt.2015.11.010 (2016).

27 Griffin, J. T. et al. Gradual acquisition of immunity to severe malaria with increasing exposure. Proceedings of the Royal Society B: Biological Sciences 282 (2015).

28 Slater, H. C., Walker, P. G. T., Bousema, T., Okell, L. C. \& Ghani, A. C. The Potential Impact of Adding Ivermectin to a Mass Treatment Intervention to Reduce Malaria Transmission: A Modelling Study. The Journal of Infectious Diseases 210, 1972-1980, doi:10.1093/infdis/jiu351 (2014).

29 Winskill, P., Slater, H. C., Griffin, J. T., Ghani, A. C. \& Walker, P. G. T. The US President's Malaria Initiative, Plasmodium falciparum transmission and mortality: A modelling study. PLOS Medicine 14, e1002448, doi:10.1371/journal.pmed.1002448 (2017).

30 Smit, M. R. et al. Pharmacokinetics-Pharmacodynamics of High-Dose Ivermectin with Dihydroartemisinin-Piperaquine on Mosquitocidal Activity and QT-prolongation (IVERMAL). Clinical Pharmacology \& Therapeutics 105, 88-401, doi:10.1002/cpt.1219 (2019).

31 Cairns, M. E. et al. Seasonality in malaria transmission: implications for case-management with long-acting artemisinin combination therapy in sub-Saharan Africa. Malaria Journal 14, 321, doi:10.1186/s12936-015-0839-4 (2015).

32 Ouédraogo, A. L. et al. Efficacy and Safety of the Mosquitocidal Drug Ivermectin to Prevent Malaria Transmission After Treatment: A Double-Blind, Randomized, Clinical Trial. Clinical Infectious Diseases 60, 357-365, doi:10.1093/cid/ciu797 (2015).

33 Fritz, M. L., Walker, E. D. \& Miller, J. R. Lethal and Sublethal Effects of Avermectin/Milbemycin Parasiticides on the African Malaria Vector, Anopheles arabiensis. Journal of Medical Entomology 49, 326-331, doi:10.1603/ME11098 (2012).

34 Richards, F. O. Mass Administration of Ivermectin in Areas Where Loa loa Is Endemic. New England Journal of Medicine 377, 2088-2090, doi:10.1056/NEJMe1712713 (2017).

35 Smit, M. R. et al. Pharmacokinetics-Pharmacodynamics of High-Dose Ivermectin with Dihydroartemisinin-Piperaquine on Mosquitocidal Activity and QT-prolongation (IVERMAL). Clinical Pharmacology \& Therapeutics 0, doi:10.1002/cpt.1219 (2018). 


\section{Box 1: Details of intervention scenarios}

\begin{tabular}{|c|c|c|c|}
\hline $\begin{array}{l}\text { Complementary } \\
\text { intervention }\end{array}$ & $\begin{array}{l}\text { Ivermectin } \\
\text { interventions }\end{array}$ & $\begin{array}{l}\text { Intervention } \\
\text { Frequency }\end{array}$ & $\begin{array}{l}\text { Example transmission } \\
\text { setting }\end{array}$ \\
\hline \multirow{3}{*}{$\begin{array}{l}\text { Continue standard } \\
\text { interventions already } \\
\text { in place (LLINs, access } \\
\text { to diagnosis and } \\
\text { treatment) }\end{array}$} & \multirow{3}{*}{$\begin{array}{ll}\text { - } & \text { None } \\
\text { - Ivermectin- } \\
\text { 1x400 MDA } \\
\text { - Ivermectin- } \\
\text { 3x300 MDA }\end{array}$} & \multirow[t]{3}{*}{$\begin{array}{l}3 \text { rounds } \\
\text { one month } \\
\text { apart }\end{array}$} & $\begin{array}{l}\text { Highly seasonal (based on } \\
\text { Fatick, Senegal), moderate } \\
\text { transmission }\end{array}$ \\
\hline & & & $\begin{array}{l}\text { Seasonal (based on } \\
\text { Bougouriba, Burkina Faso), } \\
\text { moderate transmission }\end{array}$ \\
\hline & & & $\begin{array}{l}\text { Perennial (based on } \\
\text { Equateur, DRC), moderate } \\
\text { transmission }\end{array}$ \\
\hline \multirow{2}{*}{$\begin{array}{l}\text { SMC (with sulfadoxine- } \\
\text { pyrimethamine and } \\
\text { amodiaquine (SP-AQ)) } \\
\text { to children 3-59 } \\
\text { months old and } \\
\text { continue standard } \\
\text { interventions }\end{array}$} & \multirow{2}{*}{$\begin{array}{ll}- & \text { None } \\
\text { - } & \text { Ivermectin- } \\
& \text { 1x400 MDA } \\
\text { - } & \text { Ivermectin- } \\
& 3 \times 300 \text { MDA }\end{array}$} & \multirow[t]{2}{*}{$\begin{array}{l}4 \text { rounds } \\
\text { one month } \\
\text { apart }\end{array}$} & $\begin{array}{l}\text { Highly seasonal (based on } \\
\text { Fatick, Senegal), moderate } \\
\text { transmission }\end{array}$ \\
\hline & & & $\begin{array}{l}\text { Seasonal (based on } \\
\text { Bougouriba, Burkina Faso), } \\
\text { moderate transmission }\end{array}$ \\
\hline \multirow{4}{*}{$\begin{array}{l}\text { MDA (with } \\
\text { dihydroartemisinin- } \\
\text { piperaquine (DHA-P)) } \\
\text { to all eligible } \\
\text { population and } \\
\text { continue standard } \\
\text { interventions }\end{array}$} & \multirow{4}{*}{$\begin{array}{l}\text { - } \\
\text { - Ivermectin- } \\
\text { 1x400 MDA } \\
\text { - Ivermectin- } \\
\text { 3×300 MDA }\end{array}$} & \multirow[t]{3}{*}{$\begin{array}{l}3 \text { rounds } \\
\text { one month } \\
\text { apart }\end{array}$} & $\begin{array}{l}\text { Highly seasonal (based on } \\
\text { Fatick, Senegal), moderate } \\
\text { transmission }\end{array}$ \\
\hline & & & $\begin{array}{l}\text { Perennial (based on } \\
\text { Equateur, DRC), moderate } \\
\text { transmission }\end{array}$ \\
\hline & & & $\begin{array}{l}\text { Highly seasonal (based on } \\
\text { Fatick, Senegal), low } \\
\text { transmission }\end{array}$ \\
\hline & & $\begin{array}{l}3 \text { rounds } \\
\text { four months } \\
\text { apart }\end{array}$ & $\begin{array}{l}\text { Perennial (based on } \\
\text { Equateur, DRC), moderate } \\
\text { transmission }\end{array}$ \\
\hline
\end{tabular}




\begin{tabular}{|c|c|c|c|c|c|}
\hline \multirow[b]{2}{*}{ Ivermectin dose } & \multicolumn{3}{|c|}{$\begin{array}{l}\text { Percentage reduction in clinical } \\
\text { incidence in all population over } 1 \\
\text { year after start of intervention }\end{array}$} & \multicolumn{2}{|c|}{$\begin{array}{l}\text { Percentage reduction in mean } \\
\text { slide prevalence in all population } \\
\text { over } 1 \text { year after start of } \\
\text { intervention }\end{array}$} \\
\hline & & $1 \times 400 \mu \mathrm{g} / \mathrm{kg}$ & $3 \times 300 \mu \mathrm{g} / \mathrm{kg}$ & $1 \times 400 \mu \mathrm{g} / \mathrm{kg}$ & $3 \times 300 \mu \mathrm{g} / \mathrm{kg}$ \\
\hline Highly seasonal (Fatick, Senegal) & & $62 \%$ & $71 \%$ & $27 \%$ & $34 \%$ \\
\hline Seasonal (Bougouriba, BF) & & $49 \%$ & $55 \%$ & $21 \%$ & $26 \%$ \\
\hline Perennial (Equateur, DRC) & & $28 \%$ & $31 \%$ & $13 \%$ & $15 \%$ \\
\hline \multicolumn{6}{|l|}{ SMC and ivermectin MDA } \\
\hline & \multicolumn{3}{|c|}{$\begin{array}{l}\text { Percentage reduction in clinical } \\
\text { incidence in children }<5 \text { years old } \\
\text { over } 1 \text { year after start of } \\
\text { intervention (compared to baseline) }\end{array}$} & \multicolumn{2}{|c|}{$\begin{array}{l}\text { Percentage reduction in clinical } \\
\text { incidence in children }<5 \text { years old } \\
\text { over } 1 \text { year after start of } \\
\text { intervention (compared to SMC } \\
\text { only) }\end{array}$} \\
\hline Complementary intervention & \multicolumn{5}{|c|}{ SMC to children $<5$ years old only } \\
\hline Ivermectin dose & None & $1 \times 400 \mu \mathrm{g} / \mathrm{kg}$ & $3 \times 300 \mu \mathrm{g} / \mathrm{kg}$ & $1 \times 400 \mu g / k g$ & $3 \times 300 \mu \mathrm{g} / \mathrm{kg}$ \\
\hline Highly seasonal (Fatick, Senegal) & $58 \%$ & $87 \%$ & $90 \%$ & $69 \%$ & $77 \%$ \\
\hline Seasonal (Bougouriba, BF) & $48 \%$ & $75 \%$ & $78 \%$ & $51 \%$ & $58 \%$ \\
\hline \multicolumn{6}{|l|}{ MDA with DHA-P and ivermectin } \\
\hline & \multicolumn{3}{|c|}{$\begin{array}{l}\text { Percentage reduction in clinical } \\
\text { incidence in all population over } 1 \\
\text { year after start of intervention } \\
\text { (compared to baseline) }\end{array}$} & \multicolumn{2}{|c|}{$\begin{array}{l}\text { Percentage reduction in clinical } \\
\text { incidence in all population over } 1 \\
\text { year after start of intervention } \\
\text { (compared to MDA only) }\end{array}$} \\
\hline Complementary intervention & \multicolumn{5}{|c|}{ MDA with DHA-P } \\
\hline Ivermectin dose & None & $1 \times 400 \mu \mathrm{g} / \mathrm{kg}$ & $3 \times 300 \mu \mathrm{g} / \mathrm{kg}$ & $1 \times 400 \mu \mathrm{g} / \mathrm{kg}$ & $3 \times 300 \mu \mathrm{g} / \mathrm{kg}$ \\
\hline $\begin{array}{l}\text { Highly seasonal (Fatick, Senegal) } \\
3 \text { rounds } 1 \text { month apart }\end{array}$ & $74 \%$ & $91 \%$ & $94 \%$ & $67 \%$ & $75 \%$ \\
\hline $\begin{array}{l}\text { Perennial (Equateur, DRC) - } 3 \\
\text { rounds } 1 \text { month apart }\end{array}$ & $50 \%$ & $66 \%$ & $68 \%$ & $31 \%$ & $36 \%$ \\
\hline \multirow{2}{*}{$\begin{array}{l}\text { Perennial (Equateur, DRC) - } 3 \\
\text { rounds } 4 \text { months apart }\end{array}$} & $57 \%$ & $79 \%$ & $81 \%$ & $51 \%$ & $57 \%$ \\
\hline & \multicolumn{3}{|c|}{$\begin{array}{l}\text { Percentage reduction in slide } \\
\text { prevalence in all population over } 1 \\
\text { year after start of intervention } \\
\text { (compared to baseline) }\end{array}$} & \multicolumn{2}{|c|}{$\begin{array}{l}\text { Percentage reduction in slide } \\
\text { prevalence in all population over } 1 \\
\text { year after start of intervention } \\
\text { (compared to MDA only) }\end{array}$} \\
\hline Complementary intervention & \multicolumn{5}{|c|}{ MDA with DHA-P } \\
\hline Ivermectin dose & None & $1 \times 400 \mu \mathrm{g} / \mathrm{kg}$ & $3 \times 300 \mu \mathrm{g} / \mathrm{kg}$ & $1 \times 400 \mu \mathrm{g} / \mathrm{kg}$ & $3 \times 300 \mu \mathrm{g} / \mathrm{kg}$ \\
\hline $\begin{array}{l}\text { Highly seasonal (Fatick, Senegal) } \\
3 \text { rounds } 1 \text { month apart }\end{array}$ & $60 \%$ & $82 \%$ & $86 \%$ & $55 \%$ & $64 \%$ \\
\hline $\begin{array}{l}\text { Perennial (Equateur, DRC) - } 3 \\
\text { rounds } 1 \text { month apart }\end{array}$ & $58 \%$ & $70 \%$ & $72 \%$ & $28 \%$ & $32 \%$ \\
\hline $\begin{array}{l}\text { Perennial (Equateur, DRC) - } 3 \\
\text { rounds } 4 \text { months apart }\end{array}$ & $64 \%$ & $78 \%$ & $79 \%$ & $38 \%$ & $43 \%$ \\
\hline
\end{tabular}

\section{Table 1. Predicted reductions in malaria clinical incidence and prevalence for all intervention}

scenarios described in box 1 . Ivermectin only: Percentage reductions in clinical incidence (all ages) and annual mean slide prevalence (all ages) from simulations shown in Figure 2 (BF = Burkina Faso). SMC and ivermectin MDA: Absolute and incremental (in addition to $S M C$ ) percentage reduction in clinical incidence (in children $<5$ years old), in simulations shown in Figure 3. MDA with DHA-P and ivermectin: Absolute and incremental (in addition to MDA with DHA-P) percentage reduction in clinical incidence and slide prevalence in simulations shown in Figure 4. Equations for all 'percentage reduction' equations are in the Appendix, page 10. 


\section{Slater et al, Lancet ID (in press); Confidential}

\section{Figure legends}

Figure 1: (a) Modelled drug concentrations of $3 \times 300 \mu \mathrm{g} / \mathrm{kg}$ and $1 \times 400 \mu \mathrm{g} / \mathrm{kg}$ doses of ivermectin ${ }^{35}$ estimated using a PK-PD model ${ }^{35}$ fitted to data in Smit et al. ${ }^{20}$. (b) Data (dark pink points, with $95 \%$ confidence intervals) and modelled relationship between drug concentrations and Anopheles gambiae mosquito mortality (dark pink line) obtained using local polynomial regression, and the doseresponse relationship (light pink line) used in a previous modelling study ${ }^{28}$. (c) Hazard ratios for Anopheles gambiae mosquito mortality of $3 \times 300 \mu \mathrm{g} / \mathrm{kg}$ and $1 \times 400 \mu \mathrm{g} / \mathrm{kg}$ doses of ivermectin using relationships from (a) and (b).

Figure 2: Clinical incidence per 1,000 all-age population $(a, b, c)$ and annual mean slide prevalence $(c, d, e)$ after three rounds of ivermectin one month apart in three different transmission settings. The vertical pink lines indicate the timing of the ivermectin MDA rounds. Coverage is assumed to be $70 \%$ of all individuals over the age of 5 . The baseline scenario assumes standard interventions only (LLINs, access to diagnosis and treatment).

Figure 3: Clinical incidence per 1,000 children $\leq 5$ years old after four rounds one month apart of $\mathrm{SMC}, \mathrm{SMC}+1 \times 400 \mu \mathrm{g} / \mathrm{kg}$ ivermectin or SMC $+3 \times 300 \mu \mathrm{g} / \mathrm{kg}$ ivermectin in a highly seasonal (a) and seasonal (b) transmission setting with a pre-intervention mean annual slide prevalence of $30 \%$. We assume ivermectin coverage of $70 \%$ in individuals over the age of five and SMC coverage of $90 \%$ children aged 3-59 months. The baseline scenario assumes standard interventions only (LLINs, access to diagnosis and treatment).

Figure 4: Clinical incidence per 1,000 all-age population $(a, b, c)$ and annual mean slide prevalence $(d, e, f)$ after three rounds of MDA with DHA-P, MDA with DHA-P $+1 \times 400 \mu \mathrm{g} / \mathrm{kg}$ ivermectin or MDA with DHA-P $+3 \times 300 \mu \mathrm{g} / \mathrm{kg}$ ivermectin in a highly seasonal moderate $(\mathrm{a}, \mathrm{d})$, and perennial moderate $(b, e$ and $c, f)$ transmission setting. The rounds are conducted either one month apart $(a, d$ and $b, e)$ or four months apart $(c, f)$. Coverage is assumed to be $70 \%$ of all individuals over the age of 5 . The baseline scenario assumes standard interventions only (LLINs, access to diagnosis and treatment).

Figure 5: Clinical incidence per 1,000 all-age population (a) and annual mean slide prevalence (b) after three rounds of MDA with DHA-P, MDA with DHA-P + 1x400 $\mathrm{gg} / \mathrm{kg}$ ivermectin or MDA with DHA-P + $3 \times 300 \mu \mathrm{g} / \mathrm{kg}$ ivermectin in a highly seasonal low transmission setting. Coverage is assumed to be $70 \%$ of all individuals over the age of 5 . The baseline scenario assumes standard interventions only (LLINs, access to diagnosis and treatment). 

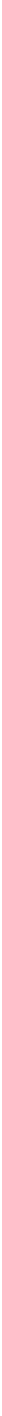

$\begin{array}{llllllllllllllllllllllll}0 & 1 & 2 & 3 & 4 & 5 & 6 & 7 & 8 & 9 & 10 & 11 & 12 & 13 & 14 & 15 & 16 & 17 & 18 & 19 & 20 & 21 & 22 & 23\end{array}$ 
Highly Seasonal Transmission

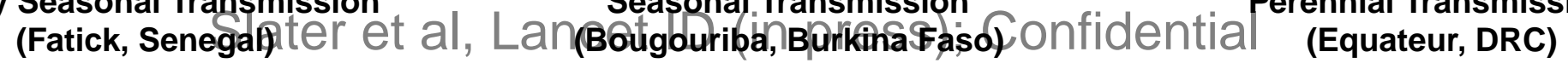

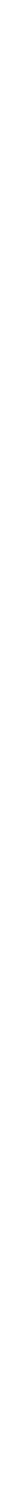


Hightly seàsoñat Transmissiont

(Fatick, Senegal)

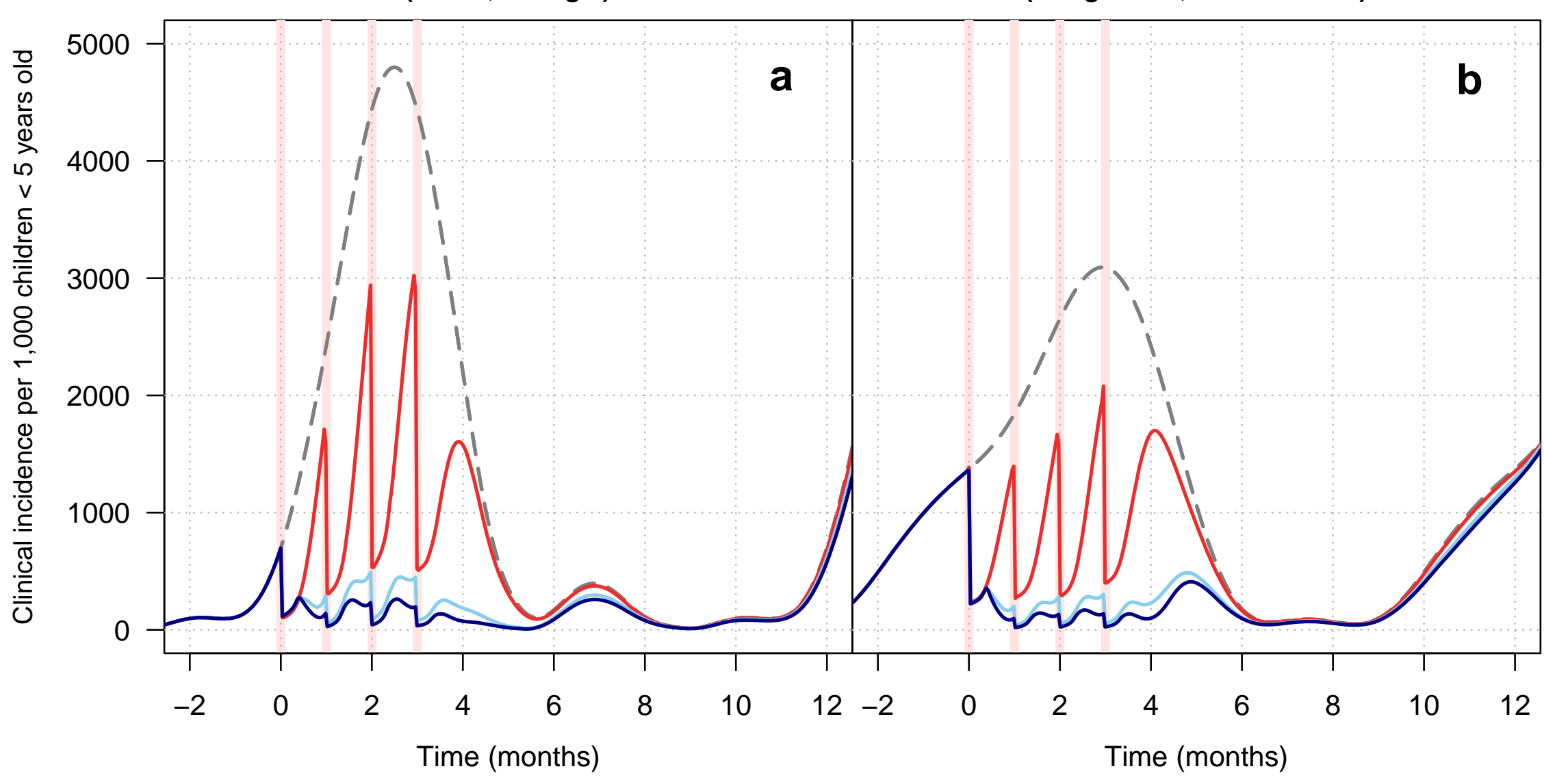

- Baseline — SMC 

(Fatick, Senegâl) et al, Lancet l(Equateur,QDRC) Confidential (Equateur, DRC)

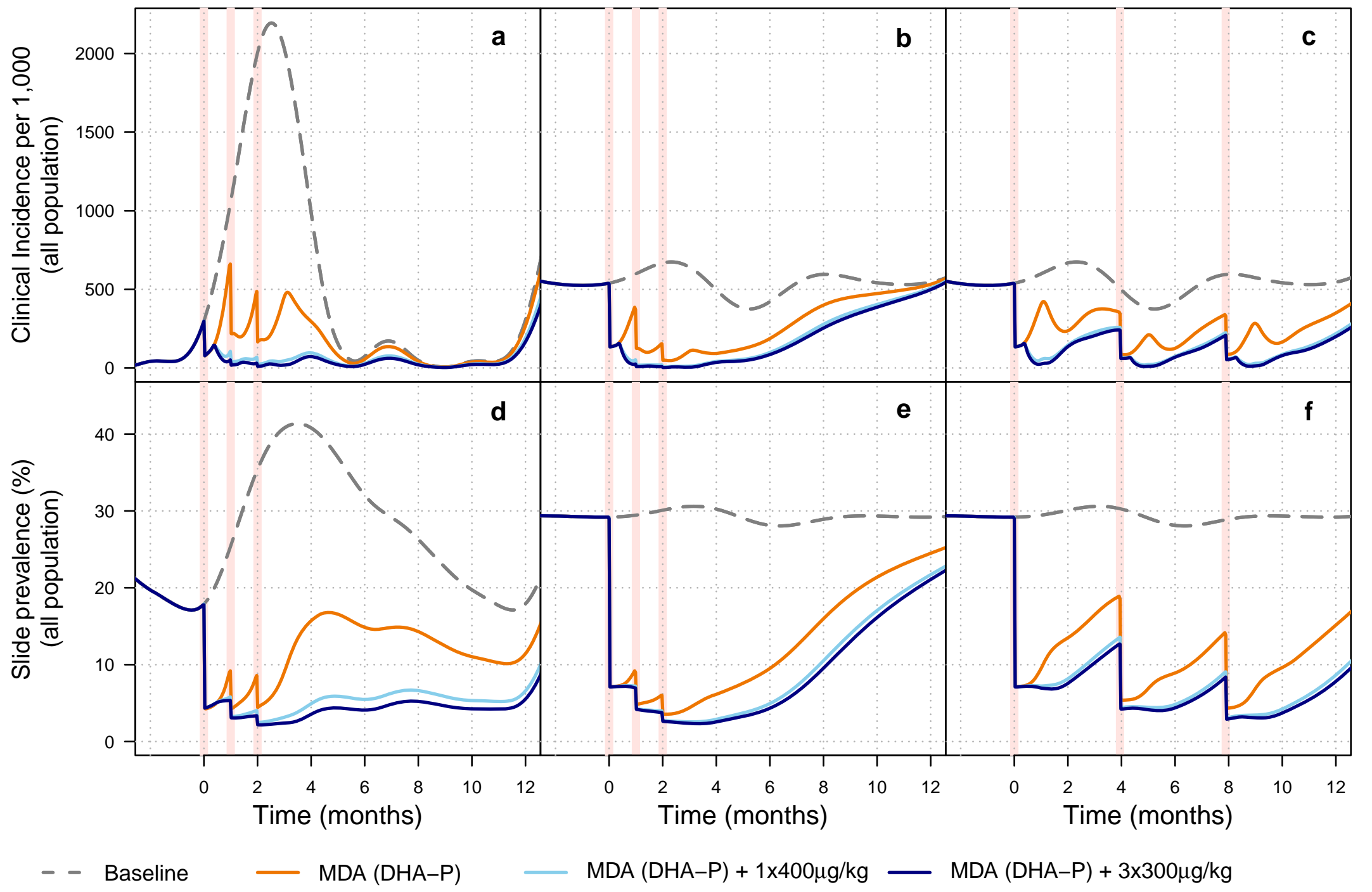


Slater et al, Lancet ID (in press); Confidential
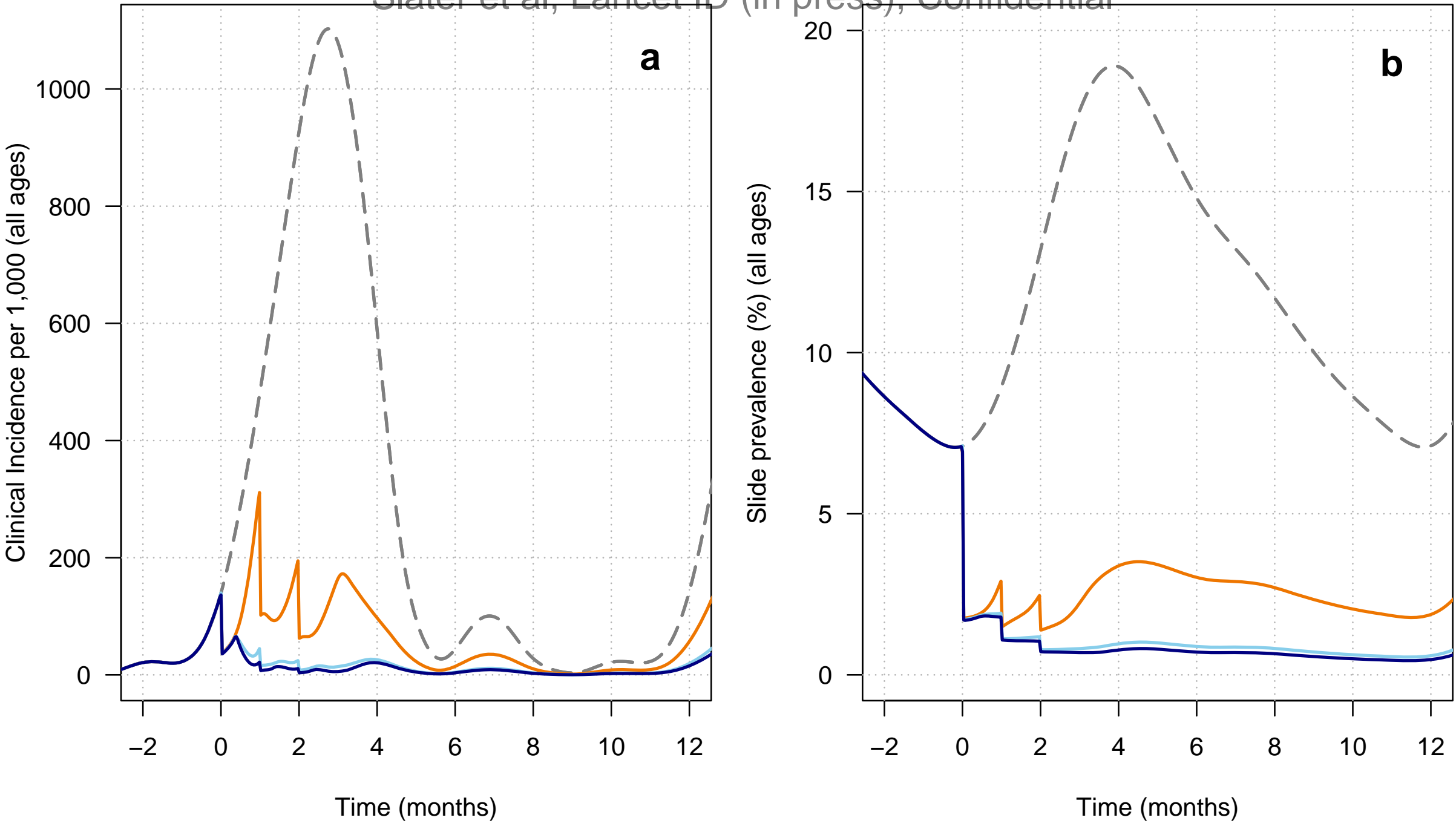

- Baseline $-\operatorname{MDA}(\mathrm{DHA}-\mathrm{P})-\mathrm{MDA}(\mathrm{DHA}-\mathrm{P})+1 \times 400 \mu \mathrm{g} / \mathrm{kg}-\mathrm{MDA}(\mathrm{DHA}-\mathrm{P})+3 \times 300 \mu \mathrm{g} / \mathrm{kg}$ 